\title{
The Algorithm-Oriented Management of Nasal Bone Fracture according to Stranc's Classification System
}

\author{
Ki-Sung Park, \\ Seung-Soo Kim, \\ Wu-Seop Lee, \\ Wan-Suk Yang \\ Department of Plastic and Reconstructive \\ Surgery, Dong Kang General Hospital, Ul- \\ san, Korea
}

This article contains supplemental Fig. S1.

No potential conflict of interest relevant to this article was reported.

\begin{abstract}
Background: Nasal bone fracture is one of the most common facial bone fracture types, and the surgical results exert a strong influence on the facial contour and patient satisfaction. Preventing secondary deformity and restoring the original bone state are the major goals of surgeons managing nasal bone fracture patients. In this study, a treatment algorithm was established by applying the modified open reduction technique and postoperative care for several years.

Methods: This article is a retrospective chart review of 417 patients who had been received surgical treatment from 2014 to 2015 . Using prepared questionnaires and visual analogue scale, several components (postoperative nasal contour; degree of pain; minor complications like dry mouth, sleep disturbance, swallowing difficulty, conversation difficulty, and headache; and degree of patient satisfaction) were evaluated.

Results: The average scores for the postoperative nasal contour given by three experts, and the degree of patient satisfaction, were within the "satisfied" (4) to "very satisfied" (5) range $(4.5,4.6,4.5$, and 4.2 , respectively). The postoperative degree of pain was sufficiently low that the patients needed only the minimum dose of painkiller. The scores for the minor complications (dry mouth, sleep disturbance, swallowing difficulty, conversation difficulty, headache) were relatively low $(36.4,40.8,65.2,32.3$, and 34 out of the maximum score of 100, respectively).

Conclusion: Satisfactory results were obtained through the algorithm-oriented management of nasal bone fracture. The degree of postoperative pain and minor complications were considerably low, and the degree of satisfaction with the nasal contour was high.
\end{abstract}

Keywords: Nasal bone / Fractures / Injuries

\section{INTRODUCTION}

Nasal bone fracture is the most frequently occurring facial bone fracture, and secondary deformity can be easily noticeable if the fracture is inappropriately treated. In many cases, especially those where it is considered a minor injury, nasal bone fracture can be treated simply and within a short time through the closed reduction (CR) technique [1]. As the CR technique has limitations, however, compared to the open reduction $(\mathrm{OR})$ technique in

\footnotetext{
Correspondence: Wan Suk Yang

Department of Plastic and Reconstructive Surgery, Dong Kang General Hospital, 239

Taewharo, Jung-gu, Ulsan 44455, Korea

E-mail: artps@naver.com

Received February 14, 2017 / Revised May 25, 2017 / Accepted May 25, 2017
}

terms of the capability for sophisticated manipulations, it is necessary to select the optimal operating technique for individuals depending on their nasal fracture pattern. If surgeons establish a proper surgical plan and apply appropriate surgical skills, they may obtain the best results in terms of the time and cost of the surgery, and of the postoperative degree of patient satisfaction [2]. To achieve a reliable diagnosis and consistency of the surgical results, it is important to classify nasal bone fracture systemically, and to treat it appropriately by employing the proper therapeutic algorithm [3].

A treatment algorithm was established according to Stranc's classification system, and some modified versions of the widely known CR technique and the OR through endonasal incision 
technique were applied. This study aimed to provide useful information about establishing the optimal treatment algorithm for nasal bone fracture by analyzing the treatment results of patients with such fracture type.

\section{METHODS}

\section{Patients and treatment algorithm}

In this study, a retrospective chart review of 417 patients who had been diagnosed with nasal bone fracture and received surgical treatment from 2014 to 2015 was conducted. The patterns of nasal fracture were classified according to Stranc's classification system [4]. Nasal fracture is classified into frontal- and lateral-impact fracture according to the direction of power, and into plane 1 , plane 2, plane 3 , and comminuted fracture according to the strength of the fracture (Table 1). The patients with frontal plane 1 fracture were treated with OR accompanied by C-arm. The patients with frontal plane 2 and lateral plane 1, 2 fracture were treated with OR through endonasal incision. For the patients with comminuted fracture, CR was conducted (Table 2, Fig. 1). For all the patients, plain radiography (Waters' view, nasal-bone lateral view from both sides) was conducted before and after the surgery, and computed tomography (CT) was carried out before the surgery to identify the fracture patterns. Postoperative CT was selectively conducted for very few patients due to insurance issues.

\section{Surgical techniques}

\section{Open reduction through endonasal incision}

Lidocaine 2\% with epinephrine 1:100,000 is locally injected at proximal $3 \mathrm{~mm}$ rather than through the internal nasal valve, under general anesthesia, for the prevention of mucosal perforation and to decrease the bleeding. Then an about- $3 \mathrm{~mm}$ incision is

Table 1. Distribution of the patients according to nasal bone fracture types Fracture type No. of patients (\%)

\begin{tabular}{lc} 
Frontal plane 1 & $72(17.3)$ \\
\hline Frontal plane 2 & $55(13.3)$ \\
Lateral plane 1 & $194(46.4)$ \\
Lateral plane 2 & $78(18.8)$ \\
Comminuted & $18(4.2)$ \\
Total & $417(100)$
\end{tabular}

Table 2. Distribution of the patients according to operation technique Operation technique No. of patients $(\%)$

Open reduction with C-arm $72(17.3)$ Open reduction through endonasal incision $\quad 327$ (78.5) Closed reduction 18 (4.2)

Total $\quad 417(100)$

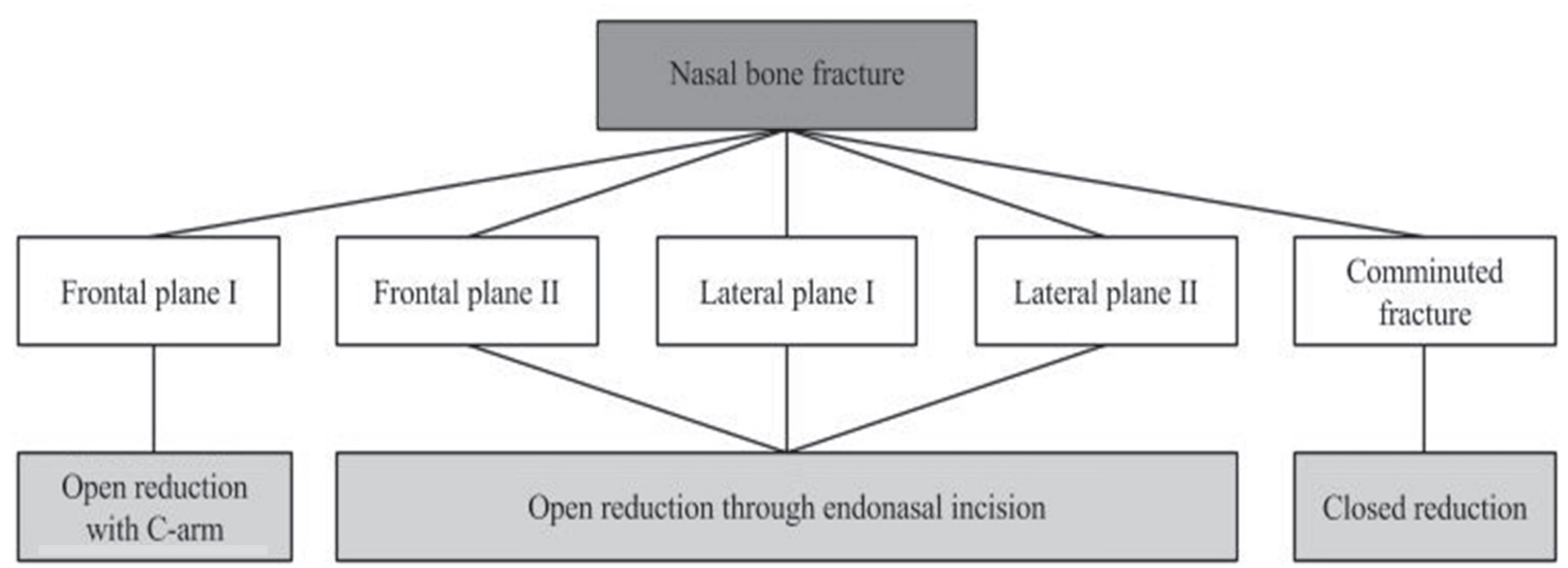

Fig. 1. The algorithm for nasal bone fracture management. 
deeply made using the No. 15 blade. The space between the periosteum and the submucosa is dissected using a mosquito clamp. After the elevator is located between the nasal bone and the mucosa, the alignment of the nasal bone on both sides is corrected using tactile sense. The deviated septum is corrected using Walsham forceps. On the opposite side of the depressed nasal bone, the protruded bone is reduced. The incision sites are sutured after checking if the bone parts of the nasal bone and septum have been properly reduced. For supporting the reduced bone, Nasopore (Forte Plus type) is packed into the superior meatus. A silicone internal nasal septal splint (Fig. 2) is inserted and anchored into the distal septum. For nasal mucous membrane protection and splint fixation, Nasopore (standard type) is inserted in the middle meatus. Finally, Thermo-Splint is applied and fixated with adhesive plaster.

\section{Open reduction with $C$-arm}

In the frontal plane 1 pattern, the injuries are restricted to the distal end of the nasal bone. The approaching method is the same as that described in OR through endonasal incision, but the manipulations focus on the injured tip of the nasal bone. Looking at the C-arm image in real time, the surgeon inserts the elevator and pulls it in an anterior (forward) direction to raise and straighten the injured nasal bone. The elevator is rotated and canted out lat-

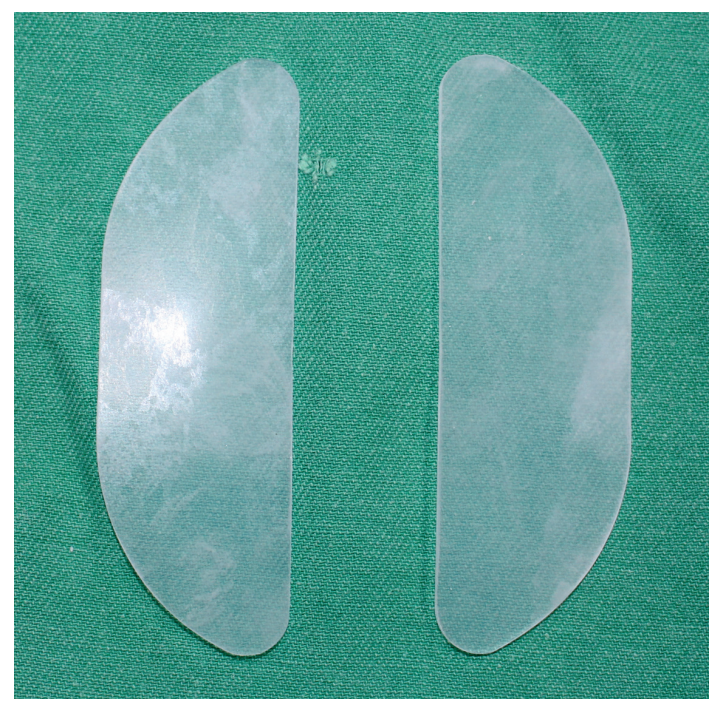

Fig. 2. Silicone internal nasal septal splint. erally several times to correct the collapsed-bone segments. After confirming that the nasal bone has been properly reduced, the incision sites are sutured, and Nasopore (Fig. 3) is directly packed under the corrected bone segments.

\section{Closed reduction}

The purpose of CR in comminuted fracture is to correct the fragmented bone segments with minimal damage to the soft tissues that support them. With no incision, instruments are inserted and manipulated carefully based on imaging study and tactile sense. Although the basic techniques of manipulation are similar to those of OR, the surgeon should exert utmost efforts to apply sufficient strength to correct the fractured bone, without unnecessary movements. In most cases of comminuted fracture, sophisticated septoplasty is necessary, and other accompanying facial bone fractures are common [5].

\section{Assessment methods}

\section{Postoperative nasal contour}

The data on the photography and plain radiography of the patients were collected one month after the surgery. Then they were

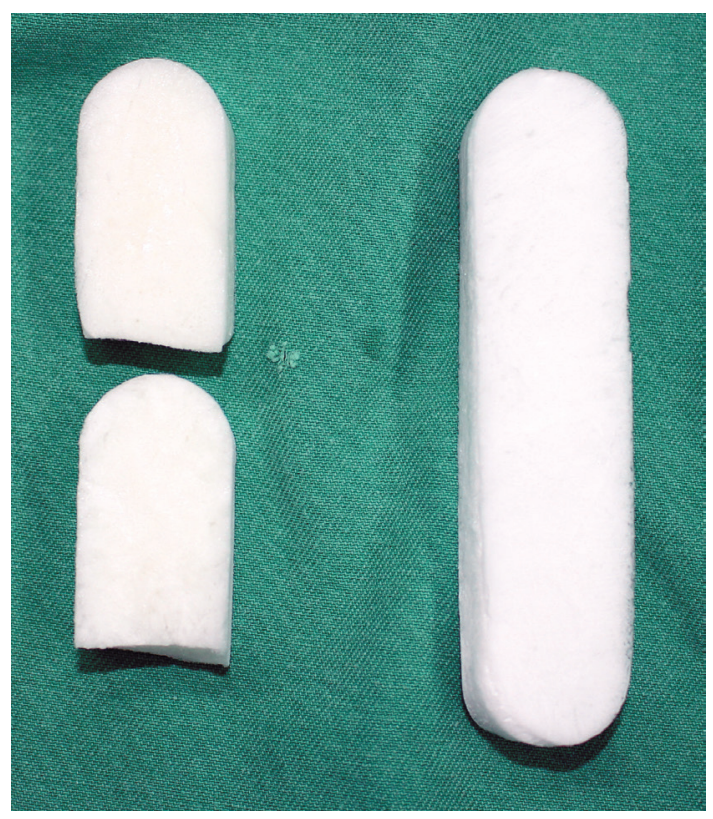

Fig. 3. Resorbable nasal packing material (Nasopore). 
compared with the preoperative data for evaluating the irregularity and deviation of the nasal bone. Three plastic surgeons were asked to choose from among the following responses to the items in the prepared questionnaire: very dissatisfied (1), dissatisfied (2), fair (3), satisfied (4), and very satisfied (5).

\section{Postoperative degree of pain}

Of the 417 patients who participated in this study, pain control analgesics (PCAs) were administered to 310 after the surgery, and were not administered to 107. For objectivity, the evaluation was conducted by dividing the patients into two groups: the group to whom PCA was administered (A) and that to whom it was not administered (B). The individual patients were asked to indicate the degree of pain that they felt on visual analogue scale (VAS) (Supplemental Fig. S1.) for five days after the surgery.

\section{Minor complications after the reduction of the nasal bone}

The patients were asked to indicate the degree of their minor complications (dry mouth, sleep disturbance, swallowing difficulty, conversation difficulty, headache) on VAS from days 1 to 5 after the surgery.

\section{Patient satisfaction}

The patients were instructed to indicate on the questionnaire the degree of their satisfaction with the treatment of their nasal bone fracture one month after the surgery. They were asked to respond to all the items in the prepared questionnaire based on the following options: very dissatisfied (1), dissatisfied (2), fair (3), satisfied (4), and very satisfied (5). To determine the difference in the degree of patient satisfaction depending on the fracture type, the patients were divided according to Stranc's classification system.

\section{Statistical analysis}

Statistical analysis was performed using SPSS ver. 22.0 (IBM Inc., ARMONK, NY, USA). Pearson's chi-square test and t-test were performed to evaluate the scores for the questionnaire. A $p$-value less than 0.05 was considered statistically significant.

\section{RESULTS}

\section{Postoperative nasal contour}

After the three plastic surgeons evaluated the data on the photography and plain radiography of changes in the patients' nasal contour (Fig. 4), the average grades given by the surgeons were 4.5, 4.6, and 4.5, respectively (Table 3). This suggests that the experts were generally satisfied with the changes in the nasal contour. There were four patients with lateral-impact plane 2, and two patients with comminuted fracture, who got grades below 2 .

\section{Postoperative degree of pain}

The day after the surgery, the grade of the pain level in the group to whom PCA had been administered was 26.4 , and that in the group to whom PCA had not been administered was 38.4 (Table 4). The patients generally required no additional painkiller and were able to endure the pain. The average grade obtained by the group to whom PCA had been administered was relatively low, and the range of score changes for five days was small.

\section{Minor complications after the reduction of the nasal bone}

For the average evaluation grades of the minor complications cited in the questionnaire until five days after the surgery, the grade of dry mouth was 36.4 , that of sleep disturbance was 40.8 , that of swallowing difficulty was 65.2 , that of conversation difficulty was 32.3, and that of headache was 34 (Table 5). As nasal stuffiness appeared in all the patients due to nasal packing, swallowing difficulty was caused in some ways, but fragmentation was gradually completed by irrigation, which showed great improvement patterns.

\section{Patient satisfaction}

The patient satisfaction score that can be used to accurately identify the shape of the nose and the degree of daily discomfort was 

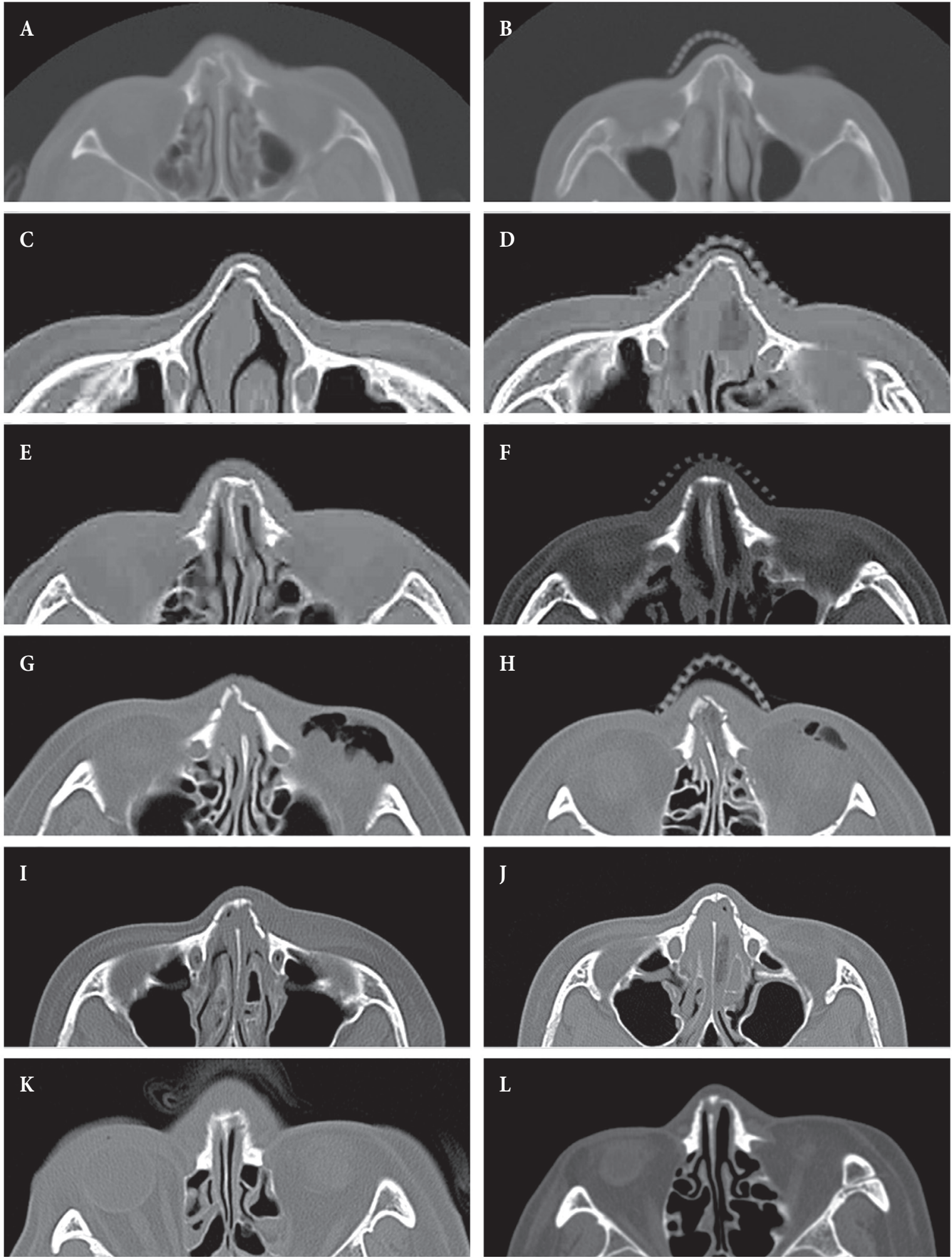

Fig. 4. Preoperative and postoperative computed tomography scan of patients. (A, B) Frontal plane 2 fracture, 3 weeks follow up. (C, D) Lateral plane 1 fracture, 2 weeks follow up. (E, F) Frontal plane 1 fracture, 3 weeks follow up. (G, H) Lateral plane 2 fracture, 1 weeks follow up (I, J) Lateral plane 1 fracture, 1 month follow up. (K, L) Comminuted fracture, 1 month follow up. 
Table 3. The satisfaction scores of postoperative nasal contour by three plastic surgeons

\begin{tabular}{lccc} 
Fracture type & A & B & C \\
\hline Frontal plane 1 & $4.8 \pm 0.58$ & $4.9 \pm 0.69$ & $4.8 \pm 0.45$ \\
\hline Frontal plane 2 & $4.4 \pm 0.26$ & $4.4 \pm 0.53$ & $4.5 \pm 0.92$ \\
\hline Lateral plane 1 & $4.6 \pm 0.66$ & $4.8 \pm 0.79$ & $4.7 \pm 0.43$ \\
Lateral plane 2 & $4.2 \pm 0.91$ & $4.4 \pm 0.85$ & $4.1 \pm 0.59$ \\
Comminuted & $3.7 \pm 0.38$ & $3.9 \pm 0.81$ & $3.8 \pm 0.74$ \\
Mean & $4.5 \pm 0.78$ & $4.6 \pm 0.69$ & $4.5 \pm 0.56$ \\
\hline
\end{tabular}

Values are mean \pm standard deviation.

A, B, C are three plastic surgeons.

Table 4. Postoperative degree of pain between PCA group and non PCA group

\begin{tabular}{lcc} 
POD & PCA $(n=310)$ & Non PCA $(n=107)$ \\
\hline 1 & $26.4 \pm 0.94$ & $38.4 \pm 0.37$ \\
2 & $12.2 \pm 0.68$ & $24.8 \pm 0.48$ \\
3 & $8.3 \pm 0.59$ & $13.2 \pm 0.51$ \\
4 & $6.7 \pm 0.47$ & $6.9 \pm 0.74$ \\
5 & $4.5 \pm 0.51$ & $5.7 \pm 0.81$ \\
\hline
\end{tabular}

Values are mean \pm standard deviation.

POD, postoperative day; PCA, pain control analgesics.

Table 5. The scores of minor complications after the reduction of nasal bone

\begin{tabular}{lccc} 
Complications & Min & Mean & Max \\
\hline Dry mouth & 19.2 & $36.4 \pm 0.74$ & 53.6 \\
Sleep disturbance & 24.6 & $40.8 \pm 0.38$ & 57.0 \\
Swollowing difficulty & 50.0 & $65.2 \pm 0.47$ & 80.4 \\
Conversation & 11.5 & $32.3 \pm 0.96$ & 53.1 \\
Headache & 23.9 & $34 \pm 0.82$ & 44.1 \\
\hline
\end{tabular}

Values are mean \pm standard deviation.

Table 6. The satisfaction scores of treatment results by patients

\begin{tabular}{lc}
\hline Fracture type & Patient satisfaction \\
\hline Frontal plane 1 & $4.5 \pm 0.88$ \\
\hline Frontal plane 2 & $4.1 \pm 0.75$ \\
\hline Lateral plane 1 & $4.4 \pm 0.68$ \\
\hline Lateral plane 2 & $3.9 \pm 0.59$ \\
\hline Comminuted & $3.5 \pm 0.73$ \\
\hline Mean & $4.2 \pm 0.85$ \\
\hline
\end{tabular}

Values are mean \pm standard deviation.

4.2 on average. Frontal plane 1 scored 4.5, frontal plane 24.1, lateral plane 14.4 , lateral plane 23.9 , and comminuted fracture 3.5 (Table 6). The higher the range and complexity of the fracture was, the lower the patient satisfaction. This is considered to be due to the higher possibility of the occurrence of nasal deformity and complications after the surgery.

\section{DISCUSSION}

Nasal bone fracture is the type of facial fracture that is most frequently presented in hospitals that have a facial trauma center [6]. Many researches on the patterns, characteristics, and operating techniques for such fracture type have been published, and modified treatment methods based on previous treatment experiences exist $[7,8]$. The closed reduction (CR) technique, which is known to have been used since a long time ago, and the indirect OR technique introduced by Burm and Oh [9] in 1998 were established as operating techniques for treating nasal bone fracture. Various classifications of nasal bone fracture have been announced, but the representative Stranc's classification system is the most widely used. Algorithms on the treatment of nasal bone fracture should be established based on appropriate classification, and treatments for individual patients should be carried out to obtain functionally and aesthetically satisfactory results after nasal bone fracture surgery.

In this study, an attempt was made to find various methods of obtaining the best results by establishing algorithms and applying treatments based on the extensive prior experience of treating patients with nasal bone fracture. Frontal plane 1 fracture was reduced relatively accurately via OR through endonasal incision while observing the movement of the nasal tip in real time using C-arm. Frontal plane 2 and lateral plane 1, 2 fracture were treated with OR through endonasal incision. In the case of comminuted fracture, the broken bone fragments that had been maintained by soft tissue can be scattered when conducting OR, and this may decrease the blood supply in the fractured bone segments [10]. For this reason, CR was conducted in this study. The range of dissection could be reduced by conducting it at an area closer to the proximal nasal bone rather than the room directly under the internal nasal valve when carrying out a local injection. After an incision was made, mucosal dissection was conducted with a mosquito clamp, and the fractured nasal bone was properly elevated 
using an elevator. Slight mucosal dissection was carried out, and subperiosteal dissection was not performed separately. The bone growth retardation that might occur after the surgery in pediatric patients was reduced by avoiding subperiosteal dissection [11].

In this study, k-wire was not used for bone fixation. The disadvantages of $\mathrm{k}$-wire are as follows: (1) as there is no separate device used for fixating the inserted wire, it may fall out; (2) perforation may occur in the frontal sinus or cribriform plate; and (3) as the bent end of the wire is exposed, it may cause inconvenience or soft-tissue damage (e.g., ulceration).

At the same time, as the nasal septum is a key factor in nasal fracture management [12], a silicone internal nasal septal splint was inserted in the nasal cavity on both sides to sufficiently support the septum after bone reduction. Also, if the need for septoplasty was identified, simultaneous correction of the septum and nasal bone was done through a joint operation with the Ear-NoseThroat Department.

For nasal packing, a resorbable nasal packing material (Nasopore) was used. This may cause less mucosal injury as it consists of relatively softer materials, and the pressure after insertion is reduced compared to a non-absorbable nasal packing material (e.g., Merocel). In addition, there is no need for removal, which may cause pain and bleeding. In this study, for about a week from the day after the surgery, saline solution was used to irrigate the nasal cavity. At this time, a sufficient amount was instructed to be used (200 mL per application, 3 times a day) to facilitate the fragmentation of the resorbable nasal packing material. This way, the inconvenience of nasal stuffiness and the feeling of irritation that the patients may experience can be resolved as quickly as possible. If necessary, suction was carried out to remove the residue. Some studies reported that such resorbable nasal packing materials might cause infection, but no such complications were observed in this study.

This study has some limitations. As the patients with nasal bone fracture were not hospitalized until a certain period of time had passed, it was difficult to do a long-term follow-up. Also, for some patients with severe comminuted fracture, nasal deformity was often observed due to the limitations of the CR technique [13]. In such cases, the necessity of conducting secondary rhino- plasty was sufficiently explained to the patients, and rhinoplasty was carried out about six months after the surgery. Also, most of the patients did not receive postoperative CT due to insurance issues.

Satisfactory results were obtained in terms of the nasal contour, the degree of patient satisfaction was found to have significantly improved, and the reported minor complications and pain were relieved. In the patient satisfaction results, the average score was within the "satisfied" to "very satisfied" range, which is considered encouraging. It remains to be seen, however, how much improvement can be attained in the "severely fractured" patient category. More sophisticated reduction can be achieved through the $\mathrm{OR}$ technique, which has various advantages over $\mathrm{CR}$. Also, nasal deformity can be reduced by finishing with a septal splint and a resorbable nasal packing material that may reduce the patient inconvenience and side effects, which will pave the way for easy postoperative management. With well-established algorithms based on prior experiences, it is obvious that surgeons can react with consistency to the corresponding treatment policy.

\section{REFERENCES}

1. Verwoerd CD. Present day treatment of nasal fractures: closed versus open reduction. Facial Plast Surg 1992;8:220-3.

2. Farber SJ, Nguyen DC, Parikh RP, Jang JL, Woo AS. Improving results in closed nasal reduction: a protocol for reducing secondary deformity. Plast Reconstr Surg 2017;139:51-9.

3. Park WY, Kim YH. A clinical study of the nasal bone fracture according to stranc classification. J Korean Soc Plast Reconstr Surg 2008;35:289-94.

4. Stranc MF, Robertson GA. A classification of injuries of the nasal skeleton. Ann Plast Surg 1979;2:468-74.

5. Kang JH, Bang YH, Lee YH, Choi CY. Clinical and statistical analysis in 452 cases of nasal bone fracture patients. J Korean Soc Plast Reconstr Surg 2011;38:775-82.

6. Murray JA, Maran AG, Mackenzie IJ, Raab G. Open v closed reduction of the fractured nose. Arch Otolaryngol 1984;110:797-802.

7. Dingman RO, Natvig P. Surgery of facial fractures Philadelphia: W.B. Saunders; 1964.

8. Rowe NL, Killey HC. Fractures of the facial skeleton. Edinburgh: E \& SLivingstone; 1970.

9. Burm JS, Oh SJ. Indirect open reduction through intercartilaginous incision and intranasal Kirschner wire splinting of comminuted nasal fractures. Plast Reconstr Surg 1998;102:342-9. 
10. Kim HS, Suh HW, Ha KY, Kim BY, Kim TY. The usefulness of the endonasal incisional approach for the treatment of nasal bone fracture. Arch Plast Surg 2012;39:209-15.

11. Wright RJ, Murakami CS, Ambro BT. Pediatric nasal injuries and management. Facial Plast Surg 2011;27:483-90.
12. Rohrich RJ, Adams WP Jr. Nasal fracture management: minimizing secondary nasal deformities. Plast Reconstr Surg 2000;106:266-73.

13. Reilly MJ, Davison SP. Open vs closed approach to the nasal pyramid for fracture reduction. Arch Facial Plast Surg 2007;9:82-6.

Supplemental Fig. S1. The visual analogue scale (VAS). It is modified version of formal 0 to 10 VAS for the purpose of evaluating components.

Supplemental data can be found at: https://e-acfs.org/src/sm/acfs-18-97-s001.pdf 